\title{
TCM Combined Chemoradiotherapy in the Treatment of Limited- Stage Small Cell Lung Cancer: Current Status and Perspectives
}

\author{
Shulin $\mathrm{He}^{1,2}$, Rui Liu${ }^{1}$, Qiujun Guo ${ }^{1,2}$, Huamin Wei, ${ }^{1,3}$, Yanjiu Bao ${ }^{1}$, Xinyao $\mathrm{Xu}^{1,2}$, Shuntai Chen ${ }^{1,2}$, \\ Honggang Zheng ${ }^{1}$ and Baojin Hua ${ }^{1 *}$
}

${ }^{1}$ Oncology Department of Guang'anmen Hospital, China Academy of Chinese Medical Sciences, Beijing, China

${ }^{2}$ Beijing University of Chinese Medicine, Beijing, China

${ }^{3}$ China Academy of Chinese Medical Sciences, Beijing, China

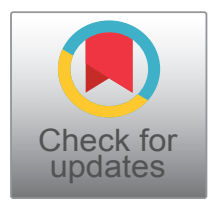

*Corresponding author: Bao Jin Hua, Oncology Department of Guang'anmen Hospital, China Academy of Chinese Medical Sciences, Beijing 100053, China

\begin{abstract}
About $13 \%$ of lung cancer cases are of the small cell subtype, but this variant is highly aggressive, and approximately $40 \%$ of patients with small cell lung cancer will have limited-stage disease, which is potentially curable with the combination of chemotherapy and radiation therapy. The standard therapy for limited-stage small cell lung cancer is concurrent chemoradiotherapy, and the 5-year survival rate observed in clinical trials is approximately $25 \%$. For patients who obtain a near complete or complete response, prophylactic cranial irradiation $(\mathrm{PCl})$ reduces the incidence of brain metastases and improves overall survival. However, chemotherapy has its limitations, the side effect caused by chemotherapy and radiotherapy is critical, so extending patient lifespan by using treatments with lower toxicities is necessary. TCM plays an important role in improving quality of life and a reduction in side effects. This may be of significant clinical value in prolonging patient's survival time.
\end{abstract}

\section{Introduction}

Lung cancer has become the most common cancer and the leading cause of cancer-related death in the world [1]. In 2010, both the incidence and mortality rates of lung cancer in China ranked first among all malignancies [2]. Lung cancer is one of the most common carcinomas not only in China, but also in the United States and Europe. It is estimated that approximately $13 \%$ of the 219,000 patients who receive a diagnosis of lung cancer will have small cell histology $[3,4]$.
Small cell lung cancer (SCLC) is characterized by rapid growth, a high prevalence of mediastinal lymph node and distant metastases at the time of diagnosis. Patients with very early stage could take the lump resection, while most patients with SCLC are not considered to be candidates for surgery. SCLC is one of the most chemosensitive solid tumors, and the outcome of SCLC patients is surely improving but slowly. Chemoradiotherapy plays a very important role in the treatment of patients with limited-stage SCLC (LS-SCLC). The chemotherapy consisting of cisplatin plus etoposide and concurrent twice-daily thoracic radiotherapy has yielded a 5 -year survival rate of approximately $25 \%$ in LS-SCLC patients $[5,6]$, which also became the standard chemoradiotherapy regimen worldwide. Prophylactic cranial irradiation (PCI) is used for patients with LS-SCLC who have achieved a complete or near complete response following initial therapy, as it decreases the risk of brain metastases and provides an overall survival benefit. However, the adverse effect caused by chemotherapy and radiotherapy is serious; TCM combined with chemotherapy demonstrated significant improvements in quality of life and a reduction in anemia and neutropenia [7]. Because of its low cost and toxicity, and high effectiveness [8,9], TCM is often used in cancer patients who have finished conventional treatment, such as surgery, chemotherapy,

Citation: He S, Liu R, Guo Q, Wei H, Bao Y, et al. (2019) TCM Combined Chemoradiotherapy in the Treatment of Limited-Stage Small Cell Lung Cancer: Current Status and Perspectives. Int J Cancer Clin Res 6:124. doi.org/10.23937/2378-3419/1410124

Accepted: September 19, 2019; Published: September 21, 2019

Copyright: (c) $2019 \mathrm{He} \mathrm{S}$, et al. This is an open-access article distributed under the terms of the Creative Commons Attribution License, which permits unrestricted use, distribution, and reproduction in any medium, provided the original author and source are credited. 
or radiotherapy and acts as a maintenance treatment. In this review, we will mainly focus on the TCM combined therapeutic options currently available for the management of the patients who have LS-SCLC at diagnosis.

\section{Disease staging}

SCLC is an aggressive disease associated with early loco-regional and distant metastases and paraneoplastic syndromes [10]. Although tumor-node-metastasis (TNM) classification has been proposed for staging SCLC $[11,12]$ It has a different staging system to that of other solid tumors [13]. In 1957, the Veterans Affairs Administration Lung Cancer Study Group (VALG) two-stage classification system introduced the terms limited disease (LD) and extensive disease (ED) [14], which is commonly used in patients with inoperable lung cancer. LS-SCLC is defined as disease that can be encompassed within a reasonable radiation field, and extensive-stage SCLC (ES-SCLC) is defined as disease that is greater than a reasonable radiation field. In 1989, the International Association for the Study of Lung Cancer (IASLC) proposed a modification of the VALSG system in which LS-SCLC was expanded to include contralateral mediastinal or supraclavicular lymph node metastases and ipsilateral pleural effusions independent of cytology [15]. ES-SCLC remained any disease at sites beyond the definition of limited disease. Current guidelines of the U.S. National Comprehensive Cancer Network (NCCN) recommend the use of positron emission tomography (PET) and CT scanning, or fused PET/CT scanning, of the chest, liver, adrenals, bone, and other areas of concern in the diagnosis and staging of SCLC.

\section{Prognostic factors}

Disease stage remains the most powerful prognostic factor for SCLC. IASLC system defines all patients without distant metastases as LS-SCLC, which criteria can help predict the prognosis and guide treatment in a better way [15]. The combination of surgery may improve the overall survival in patients with high Karnofsky performance status (KPS), tolerance to taking operation and timely getting sufficient dose of chemoradiotherapy. Some studies stated that ED, loss of weight, male patients, high PS score and tumor biomarkers are all bad prognostic factors of SCLC [16].

\section{Chemoradiotherapy and radiotherapy for limit- ed-stage small cell lung cancer}

In LS-SCLC, treatment with chemotherapy alone results in poor intrathoracic disease control, with intrathoracic failures occurring in $75 \%-90 \%$ of patients [17]. The addition of thoracic radiotherapy (TRT) to chemotherapy leads to a significantly lower rate of intrathoracic failure, to $30 \%-60 \%$. Shen jie, et al. [18] compared the effectiveness and safety of different treatment modes for LS-SCLC. They observed 171 SCLC patients who had received different therapies, 55 had received concurrent radiochemotherapy, 66 received sequential radiochemotherapy, and 50 received chemotherapy alone. For the concurrent group, sequential group, and chemotherapy alone group, the median overall survival (OS) were 30.6, 23.1, and 19.1 months, the median progression-free survival (PFS) were 19.7, 13.3, and 11.5 months, and the 5 -year survival was $28.7 \%$, $13.6 \%$, and $9.4 \%$, respectively (all $P<0.05$ ). The main toxic effects were myelosuppression, radiation pneumonia, and radiation esophagitis. The incidences of I-II grade myelosuppression were $92.7 \%, 89.4 \%$, and $92 \%$ in the concurrent group, sequential group, and chemotherapy alone group $(P=0.25)$. For concurrent group and sequential group, the incidence of I-II grade radiation pneumonia were $47.2 \%$ and $50 \%$, whereas the incidence of I-II grade radiation esophagitis were $94.5 \%$ and $75.8 \%$. Bing Xia, et al. [19] performed a quantitative analysis of tumor shrinkage due to chemotherapy and its implication for radiation treatment planning in limited-stage small-cell lung cancer, which suggests that the first cycle of chemotherapy is very important for tumor shrinkage and that initiating thoracic radiation therapy at the second cycle of chemotherapy may be a reasonable strategy for timing of radiation therapy in LS-SCLC treatment.

\section{$\mathrm{PCl}$ for limited-stage small cell lung cancer}

Despite a high response to chemotherapy and radiation therapy, most patients suffer from local recurrence or/and distant metastasis within 2 years [20]. Brain metastases are the most common site of distant failure in patients with SCLC, regardless of disease stage at presentation. The prevalence of brain metastases detected at the time of diagnosis ranges from $10 \%$ to $24 \%$ and the probability of developing brain metastases during the metastasis is associated with a poor prognosis and the median survival after the development of BM is only 4-6 months [21-23]. The positive role of $\mathrm{PCl}$ in patients with limited SCLC and extensive SCLC who achieve a complete response (CR) to chemotherapy was established in 1999 by a meta-analysis that included data from seven randomized prospective studies comparing $\mathrm{PCl}$ with no $\mathrm{PCl}$ after a CR [24]. Studies considered pivotal in supporting the use of $\mathrm{PCl}$ have been identified in three meta-analyses [25-27] and two current national guidelines [28,29].

\section{Studies in TCM combined chemotherapy}

Chemotherapy is highly responded to patients with limited disease, but chemotherapy alone is prone to induce relapse and metastases, with a high rate of loco-recurrence. The traditional treatment of SCLC is chemoradiotherapy, but the side effects are serious, which may also lead to the failure of completion of chemoradiotherapy. Traditional Chinese Medicine (TCM) has been used for thousands of years and play 
an indispensable part of alternative medicine and a vital role in adjuvant therapy of tumors [30]. Ran Wenhua, et al. [31] investigated whether TCM could improve survival rate of limited-stage small cell lung cancer patients after conventional chemoradiotherapy, 70 patients with LS-SCLC treated without surgery were randomized into two groups. The control group 35 examples treated by chemoradiotherapy, 35 cases of patients in the research group treated based on chemoradiotherapy plus TCM, which was given by mouth during strengthen phase, consolidation phase and maintenance phase. The overall 1-, 2-, 3-, 5-year survival rates of LS-SCLC patients in the control group and experimental group were $74.3 \%, 31.4 \%, 14.3 \%$, $11.4 \%$ and $77.1 \%, 40.0 \%, 28.6 \%, 22.9 \%$ respectively, and the median survival for two groups were 16.1 months and 22.3 months respectively. The difference of survival rate at years $2,3,5$ in two groups had statistically significance $(P<0.05)$. But the difference of gastrointestinal reactions and myelosuppression at I, II levels during strengthen phase in two groups had no statistically significance $(P>0.05)$. Chinese medicine treatment after conventional chemoradiotherapy can improve the survival rate and lengthen the survival time of LS-SCLC patients without increasing toxic side effects. Wang mingli, et al. [32] evaluated the efficacy of "YangYin Runfei prescription" in the treatment of small cell lung cancer using two different evaluation methods of Traditional Chinese Medicine and Western Medicine combined with serum tumor markers of small cell lung cancer Pro-GRP and NSE. After 6 courses of chemotherapy, the decline of serum markers ProGRP and NSE in combined group was more obvious than chemotherapy group $(P<0.05)$. "YangYin Runfei prescription" has attenuated synergistic effect in the treatment of small cell lung cancer.

\section{Discussion}

Small-cell lung carcinoma is an aggressive form of lung cancer that is strongly associated with cigarette smoking and has a tendency for early dissemination. Treatment of SCLC remains challenging because of its rapid growth and development of drug resistance during the course of the disease. First line chemotherapy based on cisplatin and etoposide remains the optimal treatment for LS-SCLC which increases overall survival with the same toxicity [33]. SCLC has high rate of initial response to chemotherapy (60-70\%) in patients with limited disease, but chemotherapy alone is prone to induce relapse and metastases with 5-year overall survival rate of 5\%-7\% [34,35] and loco-recurrence rate up to $80 \%[36,37]$. The traditional treatment of SCLC is chemotherapy, but the side effects are serious, which may also lead to the failure of completion of chemoradiotherapy. Based on the literature above, we found that: First, chemotherapy is the foundation in the treatment of LS-SCLC. Radiotherapy is valued for reducing local recurrence, increasing the local control rate, and improving overall survival time [38]. The earlier patients get radiotherapy, the longer the overall survival will be. Secondly, brain metastasis is one of the most important causes of treatment failure in patients with SCLC. Several randomized studies showed that PCI could reduce the rate of brain metastases in patients with SCLC who achieved CR [39]. In these studies, the rates of brain metastases were from $42.0 \%$ to $67.1 \%$ in the patients who did not experience $\mathrm{PCl}$ and $11.0 \%$ to $40.0 \%$ in the patients who experienced $\mathrm{PCl}$. For the patients with LS-SCLC who achieve CR after first-line treatment, $\mathrm{PCl}$ is an effective way in decreasing the rate of brain metastasis and tends to improve survival rate [40]. Thirdly, TCM plays an important role in multidisciplinary treatment of malignant tumors. The main mechanisms of anti-tumor include: the direct cytotoxic effect, inducing tumor cell apoptosis, inhibition of angiogenesis, and modulating autoimmunity. TCM can increase the efficacy of radiotherapy and chemotherapy, reduce the side effects, [41] and raise the quality of life. Finally, although SCLC is sensitive to chemotherapy and radiotherapy, local recurrence and distant metastasis remain the leading causes of death in patients. Moreover, higher quality randomized, double-blind, controlled clinical trials are required to get more comprehensive and objective conclusions. Regular re-examination can assist in finding the metastasis earlier, ensuring that appropriate treatment is applied in time.

\section{Acknowledgments}

Funding sources: None.

\section{References}

1. International Agency for Research on Cancer. Globocan 2012: Estimated cancer incidence, mortality and prevalence worldwide in 2012.

2. Chen $\mathrm{W}$, Zheng R, Zhang S, Zhao $\mathrm{P}$, Zeng H, et al. (2014) Annual report on status of cancer in China, 2010. Chin J Cancer Res 26: 48-58.

3. Jemal A, Siegel R, Ward E, Hao Y, Xu J, et al. (2009) Cancer statistics, 2009. CA Cancer J Clin 59: 225-249.

4. Govindan R, Page N, Morgensztern D, Read W, Tierney $R$, et al. (2006) Changing epidemiology of small-cell lung cancer in the United States over the last 30 years: Analysis of the Surveillance, Epidemiologic, and End Results database. J Clin Oncol 24: 4539-4544.

5. Takada M, Fukuoka M, Kawahara M, Sugiura T, Yokoyama A, et al. (2002) Phase III study of concurrent versus sequential thoracic radiotherapy in combination with cisplatin and etoposide for limited-stage small-cell lung cancer: results of the Japan Clinical Oncology Group Study 9104. J Clin Oncol 20: 3054-3060.

6. Turrisi AT, Kim K, Blum R, Sause WT, Livingston RB, et al. (1999) Twice-daily compared with once-daily thoracic radiotherapy in limited small-cell lung cancer treated concurrently with cisplatin and etoposide. $\mathrm{N}$ Engl J Med 340: 265-271.

7. S Chen, A Flower, A Ritchie, Liu J, Molassiotis A, et al. (2010) Oral Chinese herbal medicine (CHM) as an adjuvant treatment during chemotherapy for non-small cell lung 
cancer: a systematic review. Lung Cancer 68: 137-145.

8. Hsiao WL, Liu L (2010) The role of traditional Chinese herbal medicines in cancer therapy from TCM theory to mechanistic insights. Planta Medica 76: 1118-1131.

9. Kamyab AA, Eshraghian A (2013) Anti-Inflammatory, gastrointestinal and hepatoprotective effects of Ocimum sanctum Linn: an ancient remedy with new application. Inflammation and Allergy-Drug Targets 12: 378-384.

10. Lally BE, Urbanic JJ, Blackstock AW, Miller AA, Perry MC (2007) Small cell lung cancer: have we made any progress over the last 25 years? Oncologist 12: 1096-1104.

11. Detterbeck FC, Boffa DJ, Tanoue LT (2009) The new lung cancer staging system. Chest 136: 260-271.

12. Mirsadraee $S$, Oswal D, Alizadeh $Y$, Caulo A, van Beek E Jr (2012) The 7th lung cancer TNM classification and staging system: Review of the changes and implications. World $J$ Radiol 4: 128-134.

13. Chua YJ, Steer C, Yip D (2004) Recent advances in management of small- cell lung cancer. Cancer Treat Rev 30: 521-543.

14. Stahel RA, Ginsberg R, Havemann K, Hirsch F, Ihde D, et al. (1989) Staging and prognostic factors in small cell lung cancer: a consensus report. Lung Cancer 5: 11926.

15. Micke $P$, Faldu $\mathrm{m} \mathrm{A}, \mathrm{M}$ etz $\mathrm{T}$, Beeh $\mathrm{KM}$, Bittinger $\mathrm{F}$, et al. (2002) Staging small cell lung cancer: Veterans Administration Lung Study Group versus International Association for the Study of Lung Cancer-what limits limited disease? Lung Cancer 37: 271-276.

16. Yip D, Harper PG (2000) Predictive and prognostic factors in small cell lung cancer: current status. Lung Cancer 28 : 173-185.

17. Faivre-Finn C, Lorigan P, West C, Thatcher N (2005) Thoracic radiation therapy for limited-stage small-cell lung cancer: Unanswered questions. Clin Lung Cancer 7: 23-29.

18. Shen jie, Wang LH, Zhang FQ, Wang MZ, Li Z (2013) Comparison of effectiveness and safety of different treatment modes for LS-SCLC. Zhongguo Yi Xue Ke Xue Yuan Xue Bao 35: 343-347.

19. Xia, B, Wang JZ, Liu Q, Cheng JY, Zhu ZF (2013) Quantitative analysis of tumor shrinkage due to chemotherapy and its implication for radiation treatment planning in limited-stage small-cell lung cancer. Radiat Oncol J 8: 216.

20. H Zhu, Han A, Luo J, Li M, Shi F, et al. (2014) Risk factors for brain metastases in completely resected small cell lung cancer: a retrospective study to identify patients most likely to benefit from prophylactic cranial irradiation. Radiation oncology 9: 216.

21. Socha J, Kepka L (2012) Prophylactic cranial irradiation for small-cell lung cancer: how, when and for whom? Expert Rev Anticancer Ther 12: 505-517.

22. Seute T, Leffers P, ten Velde GP, Twijnstra A (2004) Neurologic disorders in 432 consecutive patients with small cell lung carcinoma. Cancer 100: 801-806.

23. Komaki R, Cox JD, Whitson W (1981) Risk of brain metastasis from small carcinoma of the lung related to length of survival and prophylactic irradiation. Cancer Treat Rep 65: 811-814.

24. Auperin A, Arriagada R, Pignon JP, Le Pechoux C, Gregor A, et al. (1999) Prophylactic cranial irradiation for patients with small-cell lung cancer in complete remission: a metaanalysis of individual data from 987 patients. N Engl J Med 341: 476-484.

25. Hansen HH (1973) Should initial treatment of small cell carcinoma include systemic chemotherapy and brain irradiation? Cancer Chem Rep 4: 239-241.

26. Meert AP, Paesmans M, Berghmans T, Martin B, Mascaux C, et al. (2001) Pro-phylactic cranial irradiation in small cell lung: a systematic review of the literature with metaanalysis. BMC Cancer 1: 5 .

27. Viani GA, Boin AC, Ikeda VY, Vianna BS, Silva RS, et al. (2012) Thirty years of prophylactic cranial irradiation in patients with small cell lung cancer: a meta-analysis of randomized clinical trials. Jornal Brasileiro De Pneumologia: Publicacao Oficial Da Sociedade Brasileira De Pneumologia E Tisilogia 38: 372-381.

28. National Comprehensive Cancer Network. Small cell lung cancer.

29. National Institute for Health and Care Excellence [CG121].

30. Konkimalla VB, Efferth T (2008) Evidence-based Chinese medicine for cancer therapy. Journal of Ethnopharmacology 116: $207-210$.

31. Ran wenhua (2010) Clinical reasearch of limited stage small cell lung cancer treated by Chinese medcine after chemoradiotherapy. Journal of Chongqing Medical University. 35: 1756-1757.

32. Wang mingli (2014) Multi-index evaluation of integrated traditional Chinese and Western medicine treatment of small cell lung cancer. Chin J Health Lab Tec 24: 349-354.

33. Le Péchoux C, Dhermain F, Bretel JJ, Laplanche A, Dunant A, et al. (2004) Modalities of radiotherapy in small cell lung cancer: Thoracic radiotherapy and prophylactic cerebral irradiation. Rev Pneumol Clin 60: 3S91-103.

34. Warde P, Payne D (1992) Does thoracic irradiation improve survival and local control in limited stage small cell carcinoma of the lung? a meta analysis. J Clin Oncol 10: 890-895.

35. Pignon JP, Arriagade R, Ihde DE, Johnson DH, Perry MC, et al. (1992) A meta analysis of thoracic radiotherapy for small cell lung cancer. N Engl J Med 327: 1618.

36. Perry MC, Eaton WL, Propert KJ, Ware JH, Zimmer B, et al. (1987) Chemotherapy with or without radiation therapy in limited small-cell carcinoma of the lung. $\mathrm{N}$ Engl $\mathrm{J}$ Med 316: 912-918.

37. Liu Zhanwen, Li Guoliang (1996) 90 cases of limited- stage small cell lung cancer with chemotherapy and radiotherapy. Chin J Radiat Oncol 5: 42.

38. Gauler TC, Christoph DC, Fischer J, Frickhofen N, Huber R, et al. (2013) Phase-I study of sagopilone in combinationwith cisplatin in chemotherapy naïve patientswith metastasized small-cell lung cancer. Eur J Cancer 49: 2461-2468.

39. Naidoo J, Kehoe M, Sasiadek W, Hacking D, Calvert P (2014) Prophylactic cranial irradiation in small cell lung cancer: a single institution experience. IrJ Med Sci 183: 129-132.

40. Cao KJ, Huang HY, Tu MC, Pan GY (2005) Long-term results of prophylactic cranial irradiation for limited stage small-cell lung cancer in complete remission. Chin Med J 118: $1258-1262$.

41. Chen YZ, Li ZD, Zhou N, Sun H, Li PP (2012) Longer overall survival in a patient with advanced non-small cell lung cancer treated with Chinese medicine and chemotherapy. Chin J Integr Med 18: 626-628. 